\title{
Targeted therapies for myeloproliferative neoplasms
}

\author{
Bing $\mathrm{Li}^{1,2}$, Raajit K. Rampal ${ }^{3}$ and Zhijian Xiao ${ }^{1,2^{*}}$ (D)
}

\begin{abstract}
The discovery of JAK2V617F and the demonstration that BCR-ABL-negative myeloproliferative neoplasms (MPNs) are driven by abnormal JAK2 activation have led to advances in diagnostic algorithms, prognosis and ultimately also treatment strategies. The JAK $1 / 2$ inhibitor ruxolitinib was a pivotal moment in the treatment of MPNs, representing the first targeted treatment in this field. Despite a weak effect on the cause of the disease itself in MPNs, ruxolitinib improves the clinical state of patients and increases survival in myelofibrosis. In parallel, other JAK inhibitors with potential for pathologic and molecular remissions, less myelosuppression, and with greater selectivity for JAK1 or JAK2, and the ability to overcome JAK inhibitor persistence are in various stages of development. Moreover, many novel classes of targeted agents continue to be investigated in efforts to build on the progress made with ruxolitinib. This article will discuss some of the advances in the targeted therapy in this field in recent years and explore in greater detail some of the most advanced emerging agents as well as those with greatest potential.
\end{abstract}

Keywords: Targeting therapy, Myeloproliferative neoplasms

\section{Background}

The classic $B C R-A B L 1$-negative MPNs include three different disorders, essential thrombocythemia (ET), polycythemia vera (PV), and primary myelofibrosis (PMF), and are caused by constitutive activation of the cytokine receptor/JAK2 pathway due to acquired somatic mutations in three major genes [1]. JAK2V617F is the most prevalent mutation in MPNs associated with the three disorders $(65-70 \%)$ and is present in $95 \%$ of PVs. Mutations in exon 12 of JAK2 are found in around $2 \%$ of $\mathrm{PV}$, which are negative for the JAK2V617F mutation. Mutations in the thrombopoietin receptor (MPL) gene are mainly present in 5-10\% ET and PMF. The most frequent are substitutions of the Trpytophan(W)515 residue to several other amino acids, mostly Leucine(L) or Lysine (K). The third gene found frequently mutated in MPNs is calreticulin $(C A L R)$,

\footnotetext{
*Correspondence: zjxiao@ihcams.ac.cn

'MDS and MPN Centre, Institute of Hematology and Blood Diseases Hospital, Chinese Academy of Medical Sciences \& Peking Union Medical College, 288 Nanjing Road, Tianjin 300020, China

${ }^{2}$ State Key Laboratory of Experimental Hematology, Institute of Hematology and Blood Diseases Hospital, Chinese Academy of Medical Sciences \& Peking Union Medical College, Tianjin, China

Full list of author information is available at the end of the article
}

which is affected by mutations leading to a +1 frameshift in the exon 9. As with MPL mutations, CALR mutations are associated with ET and PMF but with a higher frequency $(25-35 \%)$. CALR is not a molecule directly involved in activation of JAK2, but the new Cterminus common to all mutants allows the CALR mutants to tightly bind and activate MPL and JAK2 [2-5].

With greater understanding of the key role of JAK-STAT pathway in MPN pathogenesis, the development of the JAK $1 / 2$ inhibitor ruxolitinib was a pivotal moment in the treatment of MPN, representing the first targeted treatment in this field. Although ruxolitinib is known to have striking impacts on reduction in spleen size and reduction in symptom burden, there are still many clinical challenges encountered during the use of ruxolitinib. Therefore, novel drugs and targets are being explored as mono-or combinationtherapy in this field. This article will discuss some of the advances in the targeted therapy in this field in recent years and explore in greater detail some of the most advanced emerging agents as well as those with greatest potential.

(c) The Author(s). 2019 Open Access This article is distributed under the terms of the Creative Commons Attribution 4.0 International License (http://creativecommons.org/licenses/by/4.0/), which permits unrestricted use, distribution, and reproduction in any medium, provided you give appropriate credit to the original author(s) and the source, provide a link to the Creative Commons license, and indicate if changes were made. The Creative Commons Public Domain Dedication waiver (http://creativecommons.org/publicdomain/zero/1.0/) applies to the data made available in this article, unless otherwise stated. 


\section{JAK inhibitors \\ Type I inhibitors}

Type I inhibitors target the ATP-binding site of the JAKs under the active conformation of the kinase domain [6]. Most clinically tested inhibitors are type I. They differ in their specificity for JAK2. Many inhibitors target both JAK2 and JAK1 (ruxolitinib and momelotinib). Less frequently, they target only JAK2 (NS-018, pacritinib and fedratinib).

Ruxolitinib The oral JAK1/2 inhibitor, ruxolitinib was the first approved targeted treatment for intermediateor high-risk myelofibrosis (MF) on the basis of the results of the Controlled Myelofibrosis Study with Oral JAK Inhibitor Treatment-I (COMFORT-I) [7] and COMFORT-II [8] clinical trials and for patients with PV who are refractory to or intolerant of hydroxyurea on the basis of the results of the Randomized Study of Efficacy and Safety in Polycythemia Vera With JAK Inhibitor INCB018424 Versus Best Supportive Care (RESPONSE) [9] clinical trial.

In COMFORT-I, 309 patients were randomized to either ruxolitinib or placebo, with a $\geq 35 \%$ reduction in spleen volume seen in $41.9 \%$ treated with ruxolitinib vs. $0.7 \%$ in the placebo group. In COMFORT-II, ruxolitinib was compared with best available therapy (BAT) in 219 patients, randomized in a 2:1 ratio. Similarly, the primary end point of a reduction in spleen size $\geq 35 \%$ by week 48 was seen in $28.5 \%$ of patients treated with ruxolitinib compared with $0 \%$ in the BAT group. The EORTC-QLQ-C30 scores for symptoms relevant to patients with MF showed an improvement from baseline by week 8 and continued through to week 48, indicating significant improvement in quality of life.

Following COMFORT studies, the JUMP (JAK Inhibitor RUxolitinib in Myelofibrosis Patients) study [10] was initiated. JUMP was a phase $3 \mathrm{~b}$ expandedaccess trial for patients in countries without access to ruxolitinib outside of a clinical study and included those classified as intermediate-1 risk, a population that was not included in COMFORT studies. This study further confirmed the safety and efficacy findings from an analysis of 1144 patients with intermediate- or high-risk MF, including for those patients with intermediate-1-risk disease. Furthermore, JUMP was a global study conducted in a setting that resembles routine clinical practice. Findings from this study help guide clinicians in the management of their patients with MF.

Based on COMFORT-I and COMFORT-II clinical trials, analysis of patients treated for several years with ruxolitinb indicated a significant increase in survival in Int- 2 and high-risk MF, The survival benefit with ruxolitinib was observed irrespective of baseline anemia status or transfusion requirements at week 24 . But progression to leukemia was not significantly different $[11,12]$. It is possible that most pro-survival effects derive from its palliative anti-inflammatory effects. Further analyses will be important for exploring ruxolitinib earlier in the disease course to assess the effect on the natural history of MF.

The RESPONSE study evaluated the efficacy of ruxolitinib in PV patients who were either refractory or intolerant to hydroxyurea, and had ongoing venesection requirement and splenomegaly. Patients were randomized on a 1:1 basis between ruxolitinib and BAT with $22.7 \%$ of patients in the ruxolitinib group meeting the composite end points of HCT control and $>35 \%$ splenic volume reduction at 32 weeks, compared with $0.9 \%$ in the BAT group. In RESPONSE-2 [13], 173 PV patients again resistant or intolerant to hydroxycarbamide, but without splenomegaly, were randomized between ruxolitinib and BAT, with the primary end point of HCT control achieved in $62 \%$ in the ruxolitinib group compared with $19 \%$ treated with BAT.

In refractory or hydroxyurea-resistant ET patients, ruxolitinib offered no advantage compared with other therapies in the control of the thrombocytosis and disease complications but did alleviate general symptoms and pruritus [14]. In the other [15], which was an openlabel phase 2 trial, ruxolitinib induced a meaningful reduction in platelet levels and attenuated ET-related symptoms. These preliminary results seemed superior to historically observed results, but this study was done in the absence of a comparison with another treatment.

Overall, ruxolitinib is a well-tolerated oral treatment with approximately $25-33 \%$ of adverse effects. The main toxicities are hematological, moderate anemia that may correct with time, and thrombocytopenia, which can be very severe in high-risk MF. Middleterm toxicity is an immune suppression that may be responsible for reactivation of viral infections, particularly herpes zoster and HIV1 and bacterial infections such as pneumonia, tuberculosis reactivation and urinary tract infections [16]. Long-term monitoring will be important because ruxolitinib decreases natural killer cell functions with a potential risk of solid tumor and lymphoma development [17, 18].

Despite these clinical benefits, chronic therapy with ruxolitinib has not led to molecular or pathologic remissions in the majority of MPN patients $[7,8]$ in contrast to ABL kinase inhibitors in chronic myeloid leukemia $(\mathrm{CML})$. Moreover, hematological toxicity, which is generally ruxolitinib-dose dependent and reversible, is an issue for many patients. As such there is a significant population of PMF patients who are excluded from treatment with ruxolitinib, with most avoiding its use in patients with a platelet count of $<50 \times 10^{9} / 1$. 
Momelotinib Momelotinib (CYT38) is a JAK1/JAK2 inhibitor that has shown activity resembling ruxolitinib with respect to spleen size reduction and constitutional symptom alleviation [19-21]. Importantly, momelotinib was shown to ameliorate anemia, which is a major concern in MF. These promising results led to the opening of a phase III trial for the Momelotinib Versus Ruxolitinib in Subjects With Myelofibrosis (SIMPLIFY)-1 and 2 studies in MF. However, the results of the two clinical trials did not show a major advantage of momelotinib on ruxolitinib in $\mathrm{a} \geq 35 \%$ reduction in spleen volume at week 24, although momelotinib was associated with a decrease in transfusion requirements [22, 23].

Pacritinib Pacritinib (SB1518) is a JAK2/FLT3 inhibitor. Striking results were obtained in phase 1-2 clinical trials [24]. At week $24, a \geq 35 \%$ reduction in spleen volume determined by magnetic resonance imaging (MRI) was achieved in $23.5 \%$ of evaluable patients (4/17), a $\geq 50 \%$ spleen length reduction as measured by physical examination was achieved in $47.4 \%$ (9/19). Meanwhile, 38.9\% of evaluable patients (7/18) achieved $\mathrm{a} \geq 50 \%$ decrease in MF Quality of Life and Symptom Assessment total score. Pacritinib was administered to patients with low platelet levels, as it appeared less myelosupressive. The reasons behind this feature are unclear; they could be linked to reduced specificity for MPL/JAK2 complexes. Subsequently, two phase 3 clinical trials (PERSIST -1 and -2 ) were started with different doses of pacritinib $[25,26]$.

In PERSIST-1, 327 patients were randomly assigned to pacritinib $400 \mathrm{mg}$ once daily $(n=220)$ or BAT excluding JAK2 inhibitors until disease progression or unacceptable toxicity $(n=107)$. At week $24, \mathrm{a} \geq 35 \%$ spleen length reduction was achieved by $42(19 \%)$ patients in the pacritinib group versus five (5\%) patients in the BAT group. To compare the efficacy of pacritinib with BAT, including ruxolitinib, in patients with myelofibrosis and thrombocytopenia and determine if a lower dose of pacritinib would be safer with maintained efficacy, PERSIST-2 was designed. $311 \mathrm{~Pa}-$ tients were randomized 1:1:1 to pacritinib $400 \mathrm{mg}$ once daily, pacritinib $200 \mathrm{mg}$ twice daily, or BAT. Pacritinib twice daily led to significant improvements in $a \geq 35 \%$ spleen length reduction and 50\% reduction in TSS versus BAT. Clinical improvement in hemoglobin and reduction in transfusion burden were greatest with pacritinib twice daily. Collectively, Pacritinib can provide a treatment option for patients with myelofibrosis and baseline thrombocytopenia, for whom current treatment options are limited.

Fedratinib Fedratinib (TG101348) was assessed during the JAKARTA trials with interesting clinical results [2729]. In JAKARTA-1, 289 patients with intermediate- 2 or high-risk MF were randomly assigned to fedratinib or placebo for at least 6 consecutive 4-week cycles. The primary end point of a reduction in spleen size $\geq 35 \%$ at week 24 was achieved in $38.3 \%$ of patients in the fedratinib group, vs $1 \%$ in the placebo group. Symptom response rates at week 24 were $35.1 \%$ in the fedratinib group compared with $7 \%$ in the placebo group. To further investigate the efficacy and safety of fedratinib in patients with ruxolitinib-resistant or ruxolitinibintolerant MF, 97 patients were enrolled and received at least one dose of fedratinib in JAKARTA-2. Of 83 assessable patients, $46(55 \%)$ achieved a reduction in spleen size $\geq 35 \%$ at week 24 , suggesting that patients with ruxolitinib-resistant or ruxolitinib-intolerant myelofibrosis might achieve significant clinical benefit with fedratinib. But rare patients developed Wernicke encephalopathy, which led to its stop [29]. It was assumed that it was related to an inhibition of thiamine uptake, although fedratinib does not lead to inhibition of thiamine uptake in rats [30]. The FDA removed the clinical hold in august 2017 and clinical trials are being planned.

Ns-018 NS-018 is a JAK2/Src inhibitor that has been assessed in patients with JAK2V617F-positive MF, ET, and PV. NS-018 showed an apparent increased potency for the JAK2V617F mutant in mouse models, possibly leading to less immunosuppressive effects [31]. It was tested in MF with symptom improvement but minor impact on JAK2V617F allele burden [32].

\section{Type II inhibitors}

Type II inhibitors recognize the inactive conformation of kinases and bind to the ATP-binding pocket and to the extra 'DFG-out' pocket only accessible in the conformation of inactive kinases. The so-called 'DFG-out' pocket is created by the DFG phenylalanine adopting an 'out' conformation, which means that its side chain is out of a hydrophobic spine found in the active conformation. By exploiting supplementary binding sites contiguous to the ATP-binding pocket (the DFG- pocket), type II inhibitors can gain in specificity and hence in selectivity $[6$, 33, 34]. Importantly, the extra pocket targeted by type II inhibitors is less conserved within the kinome than the canonical ATP-binding pocket, thus promising better selectivity of the compound and minimizing adverse effects due to inhibition of unintended kinases.

Two type II JAK2 inhibitors (NVP-BBT594 and NVPCHZ868) have been developed. NVP-CHZ868 has been used in preclinical models and was very effective [35]. Both inhibitors were amenable for drug development.

\section{Ruxolitinib plus other agents}

Despite the significant improvements that have been seen with the use of ruxolitinib in the management of 
patients with MF, it is recognized that it has some limitations, which include limited disease modifying activity. The COMFORT-II study showed only a $7 \%$ median reduction in JAK-2 allele burden at 48 weeks in the ruxolitinib group [36]. It is now recognized that several of the positive effects from ruxolitinib therapy may be due to a reduction in inflammatory cytokine activity rather than targeting clonal, stem cell-derived myeloproliferation, which is the primary driver of the disease. Furthermore, the median duration of spleen response is 3 years, with approximately $50 \%$ of patients discontinuing ruxolitinib treatment by $3-5$ years either due to disease progression or intolerance. In view of these factors, many studies are currently evaluating the potential for combination therapy of novel agents given alongside ruxolitinib, to maximize its efficacy and further extend the duration of response.

\section{Ruxolitinib+Azacitidine}

Abnormalities in DNA methylation are recognized in MPN, with JAK2 mutated patients showing impaired methylation of histone subsets, due to higher affinity binding of protein arginine methyltransferase 5 (PRMT5), resulting in promotion of myeloproliferation [37] (Will be discussed in the new targeting therapy).

Encouraging preliminary results have also been recently reported from phase 1-2 trials of combination therapy with ruxolitinib and the hypomethylating agent azacitidine, in patients with MF $[38,39]$. Patients were included with an overall response rate of $69 \%$, and a > $50 \%$ palpable spleen length reduction at 24 weeks was seen in $48 \%$ of patients, higher response rates than seen with ruxolitinib monotherapy. It was also noted that $26 \%$ of the spleen reductions occurred after addition of azacitidine, adding weight to the potential synergistic effect. Interestingly, reduction in JAK2 allele burden was seen in $87 \%$ of assessable patients and a reduction in bone marrow fibrosis was also seen in $41 \%$.

\section{Ruxolitinib+ histone deacetylas (HDAC) inhibitor}

HDAC inhibitors are a class of epigenetic modulators. A phase 1 study went on to investigate combination therapy with HDAC inhibitors panobinostat and ruxolitinib. The results were encouraging, with improved tolerability and higher rates of splenic volume reduction than was seen in the COMFORT studies [40]. HDAC inhibitors are recognized as being able to downregulate JAK2 activity via inhibition of HSP-90, an ATP-dependent molecular chaperone of a large number of proteins, including JAK2 [41]. JAK2 mutated cells have been shown to be more dependent on HSP-90 for preservation of their growth and survival than normal myeloid cells.

\section{Ruxolitinib+ Bromodomain and extra-terminal (BET) inhibitor}

Recent studies in MPN patients and in preclinical MPN models have shown that MPNs, in particular MF, are characterized by a chronic state of inflammation. In addition, increased levels of circulating cytokines are linked to adverse outcome in MF [42], consistent with a key role for inflammatory signaling in MPN progression and disease maintenance. These observations provide a strong rationale to investigate underlying generegulatory mechanisms that sustain chronic inflammation in MPNs. NF-kB acts as an important inflammatory signaling node in MPN. Bromodomain containing 4 (BRD4) has been shown to transcriptionally co-activate NF-kB through recognition and direct binding to acetylated p65 [43]. BET protein function is required for pathologic transcriptional NF-kB activity in MPN. In MPN mouse models, BET protein inhibition in combination with JAK kinase inhibition lead to complete reversal of reticulin fibrosis, reduce inflammatory signaling, reduce disease burden, and delays persistence associated with JAK inhibitors $[44,45]$. These studies suggest that BET inhibition, particularly in combination with JAK kinase inhibition, should be evaluated for the ability to achieve substantive clinical benefit in MPNs patients.

\section{Ruxolitinib+ poly-ADP-ribose polymerase (PARP) inhibitor}

MPN cells contain elevated levels of reactive oxygen species (ROS) and stalled replication forks, resulting in accumulation of high numbers of toxic DNA double-strand breaks (DSBs) [46, 47]. Therefore, MPN cells survival may depend on DSB repair mechanisms [48]. PARP1 plays a central role in preventing/repairing lethal DSBs by activation of base excision repair/singlestranded DNA break repair, by stimulation of fork repair/ restart, and by mediating the back-up NHEJ (B-NHEJ) repair [49]. Accumulation of potentially lethal DSBs in MPN cells could create opportunity to eliminate these cells by targeting DNA repair mechanisms.

Nieborowska-Skorska et al. [50] found that ruxolitinib inhibited two major DSB repair mechanisms, BRCAmediated homologous recombination and DNAdependent protein kinase-mediated nonhomologous end-joining, and, when combined with PARP inhibitor olaparib, caused abundant accumulation of toxic DSBs resulting in enhanced elimination of MPN primary cells, including the disease-initiating cells from the majority of patients. Moreover, the combination of Talazoparib, ruxolitinib, and hydroxyurea was highly effective in vivo in aJAK2V617F murine model and also against JAK2V617F, CALR (del52), and MPLW515L primary MPN xenografts. It is postulated that ruxolitinib-induced deficiencies in DSB repair pathways sensitized MPN cells to synthetic lethality triggered by PARP inhibitors. 


\section{New targeting therapy PRMT5 inhibitor}

Liu et al. [37] found that JAK2V617F binds to PRMT5 with more affinity than does wild-type JAK2. These oncogenic kinases also acquired the ability to phosphorylate PRMT5, greatly impairing its ability to methylate its histone substrates, and representing a specific gainof-function that allows them to regulate chromatin modifications. PRMT5 phosphorylation was readily detected in JAK2V617F-positive patient samples, and downregulation of PRMT5 in human CD34+ cells resulted in increased colony formation and erythroid differentiation. These results indicate that phosphorylation of PRMT5 contributes to the mutant JAK2-induced myeloproliferative phenotype.

Sonderegger et al. [51] developed a potent and selective SAM-dependent inhibitor of PRMT5, CTx034. In vitro study, progenitor assays of bone marrow cells from patients with MPN showed that JAK2V617F erythropoiesis was more sensitive to CTx034 than normal erythropoiesis. In vivo studies showed normalization of spleen size and erythropoiesis, comparable to Ruxolitinib treatment. Importantly, CTx034 could eradicate the malignant clone, which is rarely achieved with Ruxolitinib. Moreover, CTx034 was well tolerated in healthy animals with no suppression of hematopoiesis. This result strengthens the therapeutic rationale for PRMT5 inhibitor in MPN.

\section{Human double minute 2 (HDM2) inhibitors}

HDM2 is an important negative regulator of p53 (promotes degradation of p53), and small-molecule inhibitors of HDM2 can trigger apoptosis in cells with intact p53 function by activating p53. Because IFN-a targets JAK2V617F progenitors in PV through activation of mitogen-activated protein kinase (MAPK) and STAT1, thereby increasing p53 transcription [52]. The combination of IFN-a with HDM2 inhibitors, which prevent the degradation of $\mathrm{p} 53$, provides an opportunity to induce p53-dependent apoptosis through disparate mechanisms. In vitro and in PDX mouse model [53, 54], HDM2 antagonist Nutlin-3 or HDM2 inhibitor RG7112, with pegylated IFN-a-2a significantly decreased MPN colony formation and preferentially eliminated $J A K 2 \mathrm{~V} 617 \mathrm{~F}$ progenitors.

The clinical candidate HDM2 inhibitor idasanutlin is currently being studied in a phase 1 trial in patients with PV or ET (NCT02407080), with a provision for adding pegylated IFN-a-2a in subjects without at least a partial remission (PR) after 3 cycles. Of note, HDM2 inhibitors can cause significant thrombocytopenia by promoting p53mediated apoptosis of megakaryocyte progenitors [55].

\section{Conclusions}

The management of patients with MPN has been revolutionized by changes stemming from important discoveries regarding molecular pathogenesis, especially the central role of the JAK-STAT pathway. After the discovery of $J A K 2 \mathrm{~V} 617 \mathrm{~F}$ and the demonstration that BCR-ABLnegative MPNs are driven by abnormal JAK2 activation, there were curative expectations for JAK inhibitors. Until now, type I ATP competitors targeting the ATP-pocket of the JH1 kinase, ruxolitinib, has remained the only approved therapy for MPNs. In particular, treatment for patients with MF and extensive splenomegaly and symptomatic burden has been significantly improved following the introduction of the ruxolitinib. However, ruxolitinib for MPNs is still largely inadequate to cope with significant challenges including reduction of mutated allele burden, reversion of fibrosis, normalization of life

Table 1 List of drugs undergoing clinical trials in MPN

\begin{tabular}{|c|c|c|c|c|}
\hline NCT Number & Drugs & Target & Phase & Status \\
\hline NCT03895112 & AVID200 & TGF- $\beta 1$ and TGF- $\beta 3$ & Phase 1 & Recruiting \\
\hline NCT03566446 & CALRLong 36 peptide & CALR & Phase 1 & Active, not recruiting \\
\hline NCT03144687 & Itacitinib, Ruxolitinib & JAK & Phase 2 & Recruiting \\
\hline NCT03075826 & SGl-110 & DNMT & Phase 2 & Recruiting \\
\hline NCT03065400 & Pembrolizumab & PD-1 & Phase 2 & Recruiting \\
\hline NCT02718300 & Parsaclisib, Ruxolitinib & 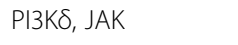 & Phase 2 & Recruiting \\
\hline NCT02493530 & TGR-1202, Ruxolitinib & 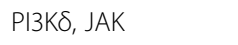 & Phase 1 & Recruiting \\
\hline NCT02407080 & RG7388, Pegasys & MDM2 & Phase 1 & Active, not recruiting \\
\hline NCT02268253 & SL-401 & CD123 & Phase1/2 & Recruiting \\
\hline NCT02257138 & Decitabine, Ruxolitinib Phosphate & DNMT, JAK & Phase $1 / 2$ & Recruiting \\
\hline NCT01761968 & Givinostat & HDAC & Phase 2 & Recruiting \\
\hline NCT01633372 & Itacitinib & JAK1 & Phase 2 & Active, not recruiting \\
\hline NCT01594723 & LY2784544 & JAK & Phase 2 & Active, not recruiting \\
\hline NCT01393509 & PU-H71 & Hsp90 & Phase 1 & Active, not recruiting \\
\hline
\end{tabular}




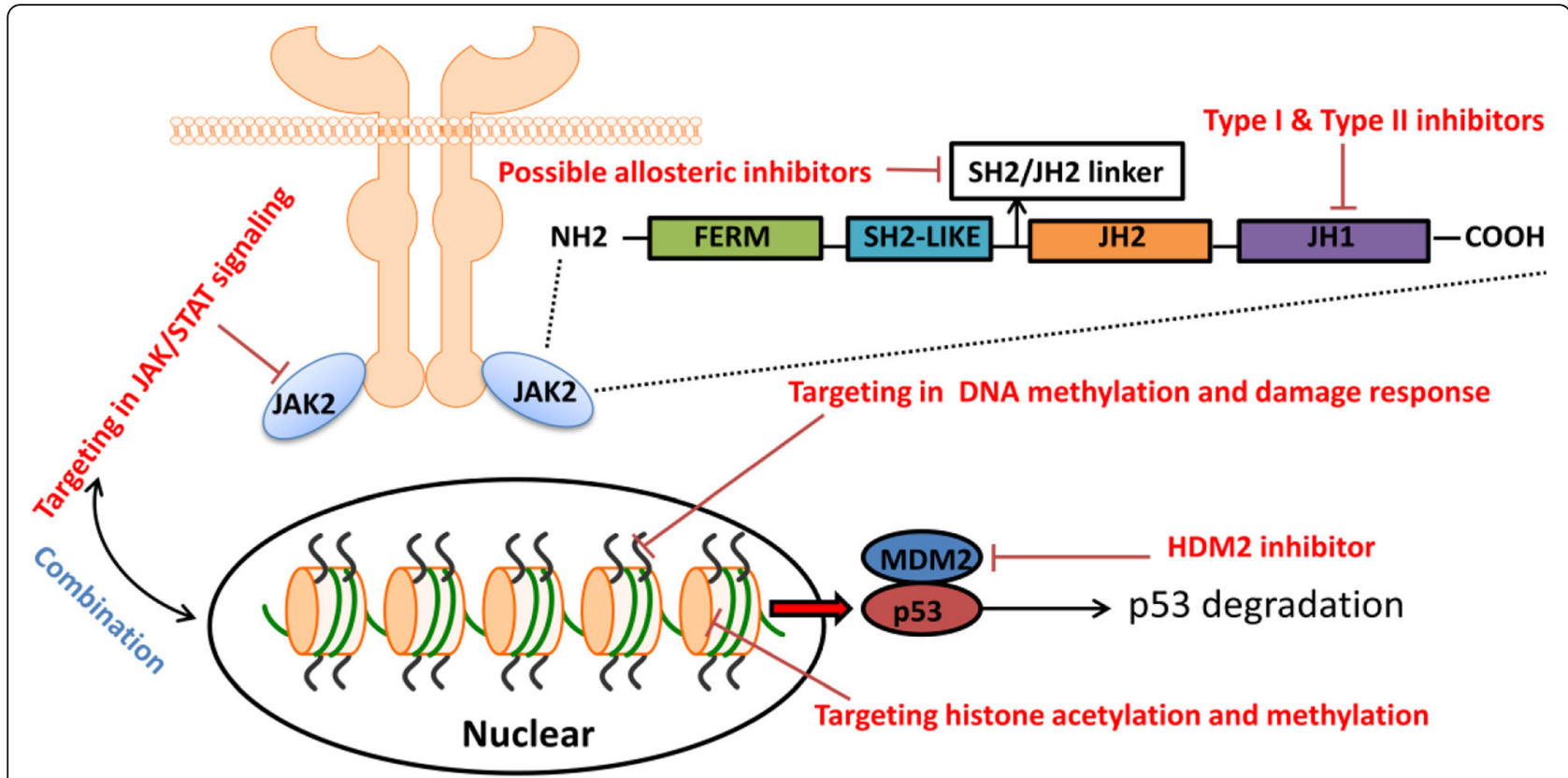

Fig. 1 The common molecular targets in MPN treatment

span and prevention of hematological progression [56]. Recently, some clinical trials of novel type I JAK inhibitors showed equal, even better effect of reduction in splenomegaly and transfusion and improvement in symptomatic burden and these results really shed light on treatment options for ruxolitinib-resistant or ruxolitinib-intolerant MPN patients. However, some of the major challenges of JAK2 inhibition: the first, to achieve potent catalytic inhibition without off-target effects; the second, to reach a high degree of selectivity for JAK2 against the other JAKs; the third, to specifically or preferentially target mutated forms against JAK2 WT, thus directly targeting the MPN clone and sparing normal hematopoiesis; finally, to specifically disrupt JAK2 function in defined cytokine receptor complexes were not overcame. Combinatorial approaches of ruxolitinib and various agents may lower toxicity and provide more effective disease control, yet more preclinical data are needed to define the most effective and synergistic combinations. In the coming decade, due to aforementioned disadvantages of ruxolitinib, the need for novel JAK inhibitors will be greater. The selectivity of JAK2, even JAK2 V617F could be achieved by developing allosteric inhibitors targeting sites outside of the active site. Allosteric inhibitors can present significant advantages compared to ATP-site binders. An allosteric site is defined as a region distinct from the kinase active site and where ligand binding can either positively or negatively regulate the enzyme activity [57]. This holds the potential of higher selectivity because every kinase possesses specific regulatory mechanisms. Moreover, targeting regions outside of the ATP-binding site will allow the development of compounds with different and improved physicochemical properties [58]. Another asset of allosteric inhibitors would be the possibility to overcome drug resistance by targeting two different sites in combination, as drug resistance usually emerges when mutations arise to prevent the compound from binding to one site [59].

Meanwhile, efforts should continue to identify other rational novel target drug targets, either alone or in combination with ruxolitinib on the basis of translational studies. Table 1 summarizes the undergoing clinical trials in MPN and Fig. 1 summarizes the common target in MPN treatment. Increased availability of next-generation sequencing and novel drugs targeting common gene mutations will go some way toward individualized targeting therapies in MPNs, especially for MF which has more complicated gene mutations than PV and ET.

\section{Abbreviations}

ATP: Adenosine triphosphate; DNA: Deoxyribonucleic acid; FDA: Food and Drug Administration; HCT: Hematocrit; PDX: Patient-Derived Xenograft

\section{Acknowledgements}

Not applicable.

Availability of data and supporting materials

Data sharing not applicable to this article as no datasets were generated or analysed in this review.

Authors' contributions

$B L, R R$ and $Z$ J wrote the typescript. All authors read and approved the final manuscript.

\section{Funding}

Supported in part by National Natural Science Funds (No.81600098,

No.81530008, No.81470297), CAMS Initiative Fund for Medical Sciences (No. 
2016-I2M-1-001), Science and technology project of Tianjin (No.15ZXLCSY00010).

\section{Ethics approval and consent to participate}

This is not applicable for this review.

\section{Consent for publication}

This is not applicable for this review.

\section{Competing interests}

The authors declare no competing financial interests.

\section{Author details}

${ }^{1}$ MDS and MPN Centre, Institute of Hematology and Blood Diseases Hospital, Chinese Academy of Medical Sciences \& Peking Union Medical College, 288 Nanjing Road, Tianjin 300020, China. ${ }^{2}$ State Key Laboratory of Experimental Hematology, Institute of Hematology and Blood Diseases Hospital, Chinese Academy of Medical Sciences \& Peking Union Medical College, Tianjin, China. ${ }^{3}$ Leukemia Service, Department of Medicine, Memorial Sloan Kettering Cancer Center, New York, USA

\section{Received: 29 May 2019 Accepted: 10 July 2019}

\section{Published online: 16 July 2019}

\section{References}

1. Vainchenker W, Kralovics R. Genetic basis and molecular pathophysiology of classical myeloproliferative neoplasms. Blood. 2017;129(6):667-79.

2. Chachoua I, Pecquet C, El-Khoury M, et al. Thrombopoietin receptor activation by myeloproliferative neoplasm associated calreticulin mutants. Blood. 2016:127(10):1325-35.

3. Lussana F, Carobbio A, Salmoiraghi S, et al. Driver mutations (JAK2V617F, MPLW515L/K or CALR), pentraxin-3 and C-reactive protein in essential thrombocythemia and polycythemia vera. J Hematol Oncol. 2017;10(1):54.

4. Elf $S$, Abdelfattah NS, Chen $\mathrm{E}_{\text {, et }}$ al. Mutant Calreticulin requires both its mutant C-terminus and the Thrombopoietin receptor for oncogenic transformation. Cancer Discov. 2016:6(4):368-81.

5. Araki M, Yang $Y$, Masubuchi $N$, et al. Activation of the thrombopoietin receptor by mutant calreticulin in CALR-mutant myeloproliferative neoplasms. Blood. 2016;127(10):1307-16.

6. Leroy $\mathrm{E}$, Constantinescu SN. Rethinking JAK2 inhibition: towards novel strategies of more specific and versatile janus kinase inhibition. Leukemia. 2017;31(5):1023-38.

7. Verstovsek S, Mesa RA, Gotlib J, et al. A double-blind, placebocontrolled trial of ruxolitinib for myelofibrosis. N Engl J Med. 2012;366(9):799-807.

8. Harrison C, Kiladjian JJ, Al-Ali HK, et al. JAK inhibition with ruxolitinib versus best available therapy for myelofibrosis. N Engl J Med. 2012;366(9):787-98.

9. Vannucchi AM, Kiladjian JJ, Griesshammer M, et al. Ruxolitinib versus standard therapy for the treatment of polycythemia vera. N Engl J Med. 2015;372(5):426-35.

10. Al-Ali HK, Griesshammer M, le Coutre P, et al. Safety and efficacy of ruxolitinib in an open-label, multicenter, single-arm phase $3 b$ expandedaccess study in patients with myelofibrosis: a snapshot of 1144 patients in the JUMP trial. Haematologica. 2016;101(9):1065-73.

11. Verstovsek S, Gotlib J, Mesa RA, et al. Long-term survival in patients treated with ruxolitinib for myelofibrosis: COMFORT-I and -II pooled analyses. J Hematol Oncol. 2017;10(1):156.

12. Verstovsek S, Mesa RA, Gotlib J, et al. Long-term treatment with ruxolitinib for patients with myelofibrosis: 5-year update from the randomized, doubleblind, placebo-controlled, phase 3 COMFORT-I trial. J Hematol Oncol. 2017; 10(1):55

13. Passamonti F, Saydam G, Lim L, et al. RESPONSE 2: a phase 3b study evaluating the efficacy and safety of ruxolitinib in patients with hydroxyurea - resistant/intolerant polycytheinia vera versus best available therapy. J Clin Oncol. 2014;32:15.

14. Harrison CN, Mead AJ, Panchal A, et al. Ruxolitinib vs best available therapy for ET intolerant or resistant to hydroxycarbamide. Blood. 2017;130(17): 1889-97.

15. Verstovsek S, Passamonti F, Rambaldi A, et al. Ruxolitinib for essential thrombocythemia refractory to or intolerant of hydroxyurea: long-term phase 2 study results. Blood. 2017;130(15):1768-71.
16. Kvasnicka HM, Thiele J, Bueso-Ramos CE, et al. Long-term effects of ruxolitinib versus best available therapy on bone marrow fibrosis in patients with myelofibrosis. J Clin Oncol. 2018;11(1):42.

17. Schonberg K, Rudolph J, Vonnahme M, et al. JAK Inhibition Impairs NK CellFunction in Myeloproliferative Neoplasms. Cancer Res 2015; 75(11): 2187-2199.

18. Curran SA, Shyer JA, St Angelo ET, et al. Human dendritic cells mitigate NKcell dysfunction mediated by nonselective JAK1/2 blockade. Cancer Immunol Res. 2017:5(1):52-60.

19. Pardanani A, Abdelrahman RA, Finke $C$, et al. Genetic determinants of responseand survival in momelotinib-treated patients with myelofibrosis. Leukemia. 2015;29(3):741-4.

20. Gupta V, Mesa RA, Deininger MW, et al. A phase 1/2, open-label study evaluating twice-daily administration of momelotinib in myelofibrosis. Haematologica. 2017;102(1):94-102.

21. Pardanani A, Gotlib JR, Gupta V, et al. Update on the long-term efficacy and safety of Momelotinib, a JAK1 and JAK2 inhibitor. For The Treatment Of Myelofibrosis Blood. 2013;122(21):108.

22. Mesa RA, Kiladjian JJ, Catalano JV, et al. SIMPLIFY-1: a phase III randomized trial of Momelotinib versus Ruxolitinib in Janus kinase inhibitor-Naïve patients with myelofibrosis. J Clin Oncol. 2017;35(34): 3844-50.

23. Harrison CN, Vannucchi AM, Platzbecker $U$, et al. Momelotinib versus best available therapy in patients with myelofibrosis previously treated with ruxolitinib (SIMPLIFY 2): a randomised, open-label, phase 3 trial. Lancet Haematol. 2018:5(2):e73-81.

24. Verstovsek $\mathrm{S}$, Odenike $\mathrm{O}$, Singer JW, et al. Phase $1 / 2$ study of pacritinib, a next generation JAK2/FLT3 inhibitor, in myelofibrosis or other myeloid malignancies. J Hematol Oncol. 2016;9(1):137.

25. Mascarenhas J, Hoffman R, Talpaz M, et al. Pacritinib vs best available therapy, including Ruxolitinib, in patients with myelofibrosis: a randomized clinical trial. JAMA Oncol. 2018:4(5):652-9.

26. Mesa RA, Vannucchi AM, Mead A, et al. Pacritinib versus best available therapy for the treatment of myelofibrosis irrespective of baseline cytopenias (PERSIST-1): an international, randomised, phase 3 trial. Lancet Haematol. 2017:4(5):e225-36.

27. Harrison CN, Schaap N, Vannucchi AM, et al. Janus kinase-2 inhibitor fedratinib in patients with myelofibrosis previously treated with ruxolitinib (JAKARTA-2): a single-arm, open-label, non-randomised, phase 2, multicentre study. Lancet Haematol. 2017;4(7):e317-24.

28. Jamieson C, Hasserjian R, Gotlib J, et al. Effect of treatment with a JAK2selective inhibitor, fedratinib, on bone marrow fibrosis in patients with myelofibrosis. J Transl Med. 2015:13:294.

29. Pardanani A, Harrison C, Cortes JE, et al. Safety and efficacy of Fedratinib in patients with primary or secondary myelofibrosis: a randomized clinical trial. JAMA Oncol. 2015;1(5):643-51.

30. Hazell AS, Afadlal S, Cheresh DA, et al. Treatment of rats with the JAK-2 inhibitor fedratinib does not lead to experimental Wernicke's encephalopathy. Neurosci Lett. 2017;642:163-7.

31. Nakaya Y, Shide K, Niwa T, et al. Efficacy of NS-018, a potent and selective JAK2/Src inhibitor, in primary cells and mouse models of myeloproliferative neoplasms. Blood Cancer J. 2011;1(7):e29.

32. Verstovsek S, Talpaz M, Ritchie E, et al. A phase I, open-label, doseescalation, multicenter study of the JAK2 inhibitor NS-018 in patients with myelofibrosis. Leukemia. 2017;31(2):393-402.

33. Blanc J, Geney R, Menet C. Type II kinase inhibitors: an opportunity in cancer for rational design. Anti Cancer Agents Med Chem. 2013;13(5):731-47.

34. Andraos R, Qian Z, Bonenfant D, et al. Modulation of activation-loop phosphorylation by JAK inhibitors is binding mode dependent. Cance Discov. 2012:2(6):512-23.

35. Meyer SC, Keller MD, Chiu S, et al. CHZ868, a type II JAK2 inhibitor, reverses type I JAK inhibitor persistence and demonstrates efficacy in Liu F, Zhao X. Myeloproliferative Neoplasms Cancer Cell. 2015;28(1):15-28.

36. Harrison CN, Vannucchi AM, Kiladjian JJ, et al. Long-term findings from COMFORT-II, a phase 3 study of ruxolitinib vs best available therapy for myelofibrosis. Leukemia. 2016;30(8):1701-7.

37. Liu F, Zhao X, Perna F, et al. JAK2V617F-mediated phosphorylation of PRMT5 downregulates its methyltransferase activity and promotes myeloproliferation. Cancer Cell. 2011;19(2):283-94.

38. Daver N, Pemmaraju N, Jabbour EJ, et al. Ruxolitinib in combination with 5azacytidine as therapy for patients with myelofibrosis. Blood. 2016;128:1127. 
39. Masarova L. Verstovsek, Hidalgo-Lopez JE, et al. a phase 2 study of ruxolitinib in combination with azacitidine in patients with myelofibrosis. Blood. 2018;132(16):1664-74.

40. Harrison CN, Heidel FH, Vannucchi AM. Efficacy, safety, and confirmation of the recommended phase 2 starting dose of the combination of ruxolitinib and panobinostat in patients with myelofibrosis. Blood. 2015;126:4060.

41. Fiskus W, Verstovsek S, Manshouri T, et al. Heat shock protein 90 inhibitor is synergistic with JAK2 inhibitor and overcomes resistance to JAK2-TKI in human myeloproliferative neoplasm cells. Clin Cancer Res. 2011;17(23): 7347-58.

42. Tefferi A, Vaidya R, Caramazza D, Finke C, Lasho T, Pardanani A. Circulating interleukin (IL)-8, IL-2R, IL-12, and IL-15 levels are independently prognostic in primary myelofibrosis: a comprehensive cytokine profiling study. J Clin Oncol. 2011;29(10):1356-63.

43. Huang B, Yang XD, Zhou MM, Ozato K, Chen LF. Brd4 coactivates transcriptional activation of NF-kappaB via specific binding to acetylated RelA. Mol Cell Biol. 2009:29(5):1375-87.

44. Sashida G, Wang C, Tomioka T, et al. The loss of Ezh2 drives the pathogenesis of myelofibrosis and sensitizes tumorinitiating cells to bromodomain inhibition. J Exp Med. 2016;213(8):1459-77.

45. Kleppe M, Koche R, Zou L, et al. Dual targeting of oncogenic activation and inflammatory signaling increases TherapeuticEfficacy in myeloproliferative neoplasms. Cancer Cell. 2018;33(4):785-7.

46. Marty C, Lacout C, Droin N, et al. A role forreactive oxygen species in JAK2 V617F myeloproliferative neoplasm progression. Leukemia. 2013; 27(11):2187-95.

47. Chen E, Ahn JS, Massie CE, et al. JAK2V617F promotes replication fork stalling with diseaserestricted impairment of the intra-S checkpoint response. Proc Natl Acad Sci U S A. 2014;111(42):15190-5.

48. Viale A, De Franco F, Orleth A, et al. Cell-cycle restriction limits DNA damage and maintains selfrenewal of leukaemia stem cells. Nature. 2009;457(7225):51-6.

49. Pilié PG, Tang C, Mills GB, Yap TA. State-of-the-art strategies for targeting the DNA damage response in cancer. Nat Rev Clin Oncol. 2019;16(2):81-104.

50. Nieborowska-Skorska M, Maifrede S, Dasgupta Y, et al. Ruxolitinib-induced defects in DNA repair cause sensitivity to PARP inhibitors in myeloproliferative neoplasms. Blood. 2017;130(26):2848-59.

51. Sonderegger S, Cerruti L, Tremblay C, et al. Smaller molecular inhibition of PRMT5 induces translational stress and p53 in JAK2V617F mutant myeloproliferative neoplasms. Blood. 2018;132:53.

52. Masarova L, Yin CC, Cortes JE, et al. Histomorphological responses after therapy with pegylated interferon a-2a in patients with essential thrombocythemia (ET) and polycythemia vera (PV). Exp Hematol Oncol. 2017;6(1):30.

53. Lu M, Wang $X$, Li $Y$, et al. Combination treatment in vitro with Nutlin, a smallmolecule antagonist of MDM2, and pegylated interferon-alpha 2a specifically targets JAK2V617F-positive polycythemia vera cells. Blood. 2012; 120(15):3098-105

54. Lu M, Xia L, Li Y, Wang X, Hoffman R. The orally bioavailable MDM2 antagonist RG7112 and pegylated interferon alpha 2a target JAK2V617Fpositive progenitor and stem cells. Blood. 2014;5(124):771-9.

55. lancu-Rubin C, Mosoyan G, Glenn K, Gordon RE, Nichols GL, Hoffman R. Activation of p53 by the MDM2 inhibitor RG7112 impairs thrombopoiesis. Exp Hematol. 2014;42:45e5.

56. Talpaz M, Erickson-Viitanen S, Hou K, Hamburg S, Baer MR. Evaluation of an alternative ruxolitinib dosing regimen in patients with myelofibrosis: an open-label phase 2 study. J Hematol Oncol. 2018;11(1):101.

57. Zhang J, Yang PL, Gray NS. Targeting cancer with small molecule kinase inhibitors. Nat Rev Cancer. 2009;9(1):28-39.

58. Cowan-Jacob SW, Mobitz H, Fabbro D. Structural biology contributions to tyrosinekinase drug discovery. Curr Opin Cell Biol. 2009;21(2):280-7.

59. Cowan-Jacob SW, Jahnke W, Knapp S. Novel approaches for targeting kinases: allosteric inhibition, allosteric activation and pseudokinases. Future Med Chem. 2014;6(5):541-61.

\section{Publisher's Note}

Springer Nature remains neutral with regard to jurisdictional claims in published maps and institutional affiliations.

\section{Ready to submit your research? Choose BMC and benefit from:}

- fast, convenient online submission

- thorough peer review by experienced researchers in your field

- rapid publication on acceptance

- support for research data, including large and complex data types

- gold Open Access which fosters wider collaboration and increased citations

- maximum visibility for your research: over $100 \mathrm{M}$ website views per year

At BMC, research is always in progress.

Learn more biomedcentral.com/submissions 